\title{
Propiedades psicométricas de una escala para medir apoyo social percibido en pacientes chilenos con diabetes tipo $2^{*}$
}

\section{Psychometric Properties of a Scale Designed to Measure Perceived Social Support in Chilean Patients with Type 2 Diabetes}

Recibido: agosto 27 de 2009 ～Revisado: marzo 14 de 2010 | Aceptado: octubre 2 de 2010

\author{
MANUel OrTiz PARAdA ** \\ MARÍA JOSÉ BAEZA RIVERA \\ Universidad de La Frontera, Temuco, Chile
}

Para citar este artículo. Ortíz, M., \& Baeza, M. J. (2011). Propiedades psicométricas de una escala para medir apoyo social percibido en pacientes chilenos con diabetes tipo 2. Universitas Psychologica, 10(1), 189-196.

\footnotetext{
Agradecimientos. Esta investigación fue financiada por el Fondo Nacional de Investigación y Desarrollo en Salud de Chile FONIS. Proyecto SA07I20071

"Factores psicosociales asociados a la adherencia al tratamiento de pacientes diabéticos tipo 2 de la ciudad de Temuco", cuyo investigador responsable es el primer autor.

*** Avenida Francisco Salazar 01145. Temuco, Chile. E-mails:msortiz@ufro.clymariajosebaezarivera@ gmail.com
}

RESUMEN

Este estudio tuvo por objetivo estimar las propiedades psicométricas de la Escala de Apoyo Social Percibido (Multidimensional Scale of Perceived Social Support [MSPSS]) de Zimet, Dahlem, Zimet y Farley, en una muestra de 76 pacientes diabéticos tipo 2, pertenecientes a la ciudad de Temuco, Chile. La escala total presenta una consistencia interna de .0849. Se condujo un análisis factorial exploratorio con rotación Varimax, obteniéndose los tres factores propuestos por los autores, que en conjunto explican el 66.8\% de la varianza. Los resultados alcanzados sugieren que esta escala posee adecuadas propiedades psicométricas para su utilización en población de pacientes con Diabetes Mellitus tipo 2.

Palabras claves autores

Análisis factorial exploratorio, consistencia interna, Escala Multidimensional de Percepción de Apoyo Social, Diabetes Mellitus.

Palabras claves descriptores

Diabetes, análisis factorial, psicometría.

\footnotetext{
A B S T R A C T

The present study tested the psychometric properties of the Zimet et al.' Multidimensional Scale of Perceived Social Support (MSPSS), in patients with Type 2 Diabetes Mellitus (DM) $(n=76)$ from Temuco City, Chile. The total scale shown appropriate levels of internal consistency $(0,849)$. An exploratory factorial analysis, with Varimax rotation, was performed over the MSPSS measures. In agreement with the original Scale, three factors were obtained explaining $66,8 \%$ of the variance. These results suggest that MSPSS is a psychometrically sound instrument that can be applied to patients with Type 2 Diabetes.

Key words authors

Exploratory Factorial Analysis, Internal Consistency, Multidimensional Scale Of

Perceived Social Support, Diabetes Mellitus.

Key words plus

Diabetes, Factor Analysis, Statistical, Psychometrics.
} 
La Diabetes Mellitus (DM) es una de las llamadas "enfermedades adquiridas" que dan cuenta del cambio en el perfil epidemiológico de la población mundial. Si bien es cierto que hace varios años las principales causas de mortalidad correspondían a enfermedades infecciosas, actualmente, son las enfermedades crónicas las que se alzan como las de mayor prevalencia e incidencia. De estas enfermedades, la diabetes mellitus tipo 1 (DM1) es una de las más frecuentes de la niñez y de la adolescencia (Kyngäs, 2000). Asimismo, la diabetes tipo 2 (DM2) se considera una de las más comunes en Europa y en Estados Unidos (Passa, 2002). Del total de diagnósticos de DM, más de un 90\% de estos corresponde a la DM2. Las estimaciones de la Organización Mundial de la Salud (OMS, 2004) señalan que para el año 2025, se espera que la de DM afecte a 300 millones de habitantes, a nivel mundial.

Ahora bien, los progresos científicos en el área de la salud permiten contar con tratamientos cada vez más efectivos. En particular, el tratamiento actualmente disponible para la DM2, bien conducido, permite disminuir la progresión de la enfermedad, aminorar sus complicaciones asociadas y elevar la calidad de vida del paciente. Sin embargo, esto resulta paradójico, pues si bien se ha avanzado en la disposición de tratamientos más efectivos, los resultados de tales tratamientos son desalentadores debido principalmente a los bajos niveles de adherencia que tiene el tratamiento de la DM2 (Quirós-Morales \& Villalobos-Pérez, 2007). Esto parece ser un problema universal, sin que aún los expertos puedan predecir adecuadamente quiénes podrían ser adherentes y quienes no (Holguín, Correa, Arrivillaga, Cáceres \& Varela, 2006).

Lo anterior podría atribuirse a la multiplicidad de factores que están en la base del comportamiento adherente, así como también la multidimensionalidad del tratamiento y la relativa independencia de los comportamientos requeridos para el tratamiento de la Diabetes Mellitus (Ortiz, 2004; Ortiz $\&$ Ortiz, 2007). De esta manera es posible que un paciente adhiera adecuadamente a la farmacoterapia, sin que esto garantice, necesariamente, el cumplimiento de las recomendaciones nutricionales o la práctica regular de actividad física.

De los factores psicosociales que más han recibido atención en el estudio del comportamiento adherente, se encuentra la percepción de apoyo social. Existe evidencia que señala que la percepción de apoyo social es una de las variables que mejor predice la adhesión al tratamiento de la DM2 (Göz, Karaoz, Goz, Ekiz \& Cetin, 2007). En un metaanálisis realizado por Di Matteo (2004), con 29 estudios acerca de la relación entre apoyo social y adherencia al tratamiento de enfermedades crónicas, se demuestra una fuerte relación entre estas variables, con diferencias de tamaño de efecto de 0,65 entre pacientes que reciben y no reciben apoyo para sus tratamientos. La estimación del odds ratio de adherencia (comparado con la no adherencia) fue de 3,6 veces mayor para sujetos que recibieron apoyo social versus los que no recibieron.

Se ha informado que el apoyo social se relaciona con conductas pilares del tratamiento de la DM2. Específicamente, pacientes diabéticos tipo 2 que demuestran tener bajos niveles de apoyo social, tienen un peor estado de salud y de bienestar general, además de presentar presión arterial alterada (Westaway, Seager, Rheeder \& Van Zyl, 2005). Se ha estimado que aquellos pacientes DM2 que tienen bajos niveles de apoyo social, tienen un 55\% más de probabilidades de fallecer que aquellos con moderados niveles de apoyo social. A su vez, estos presentan un 55\% más de probabilidades de morir que aquellos que gozan de altos niveles de apoyo social (Zhang, Norris, Gregg \& Beckles, 2007).

Debido al importante rol que parece desempeñar la percepción de apoyo social en la adherencia al tratamiento de la DM2, parece necesario contar con instrumentos válidos y confiables que permitan identificar adecuadamente la presencia de este valioso recurso en pacientes diabéticos tipo 2 . Disponer de medidas válidas y confiables de apoyo social para pacientes DM2, permitiría identificar las figuras que el paciente considera le apoyan, de modo tal de prever su potencial incorporación al tratamiento de la DM2, así como también estimu- 
lar recursos personales del paciente, en caso que tal percepción de apoyo social sea deficiente.

Existen diversos instrumentos que miden apoyo social. El SSQ, Social Support Questionnaire (Sarason, 1983), identifica a las personas a quienes con mayor frecuencia se recurre en situaciones específicas y la satisfacción con el apoyo social, tanto en áreas de sentimientos, consejo, ayuda material, retroalimentación, ayuda física y participación social. El Inventory of Socially Supportive Behaviours (ISSB) (Barrera \& Ainlay, 1983), evalúa el tipo de apoyo social recibido en sus dimensiones emocional, instrumental, informativo y social, no especificando la fuente de donde proviene. Otro instrumento es The Medical Outcomes Study Social Support Survey (MOS-SSS) (Sherbourne \& Stewart, 1991), que contempla el apoyo estructural y el funcional, evaluando 5 dimensiones: emocional, informativa, tangible, afectiva y la interacción social positiva. Estos instrumentos, si bien han sido ampliamente utilizados en estudios epidemiológicos, centran su atención más bien en los tipos de apoyo social provistos, más que en la percepción sujeto (Calvo Francés \& Díaz Palarea, 2004).

La Escala Multidimensional de Apoyo Social (Multidimensional Scale of Perceived Social Support [MSPSS]) de Zimet, Dahlem, Zimet \& Farley (1988), posee ciertas ventajas tales como haber sido diseñada específicamente para evaluar la percepción de apoyo social de tres fuentes diferentes: familia, grupo de pares y otros significativos, contando además con una subescala para cada una de estas fuentes. Diversos estudios han demostrado que esta escala posee un excelente comportamiento psicométrico (Canty-Mitchell \& Zimet, 2000; Dahlem, Zimet \& Walker, 1991; Kazarian \& McCabe, 1991). Por último, mientras algunas escalas que evalúan apoyo social o percepción del mismo son engorrosas en su aplicación, la MSPSS es una escala de autorreporte breve y de fácil utilización.

Por tanto, el objetivo de este estudio fue estimar las propiedades psicométricas de la Escala Multidimensional de Percepción de Apoyo Social de Zimet et al. (1988).

\section{Método}

\section{Participantes}

Se seleccionaron, por medio de un muestreo no probabilístico, a 76 pacientes DM2 voluntarios, usuarios del consultorio Miraflores de la Ciudad de Temuco, que cumplieran con los siguientes criterios de inclusión: a) diagnóstico de DM2 con mínimo de un año y b) pacientes usuarios del sistema público de salud. Se excluyeron aquellos sujetos que presentaron alguna comorbilidad no asociada a la DM2, tales como asma bronquial y enfermedad pulmonar obstructiva crónica.

El promedio de edad de los participantes fue 64.57 años $(D E=10.59)$. La edad promedio de los hombres fue $65.52(D E=10.49)$ y las mujeres $63.86(D E=10.74)$. El promedio de años con el diagnóstico fue 9.31 años $(D E=6.23)$. Un 43.4\% de los participantes corresponde al género masculino. El 72.3\% de la muestra está casado. En promedio los participantes tienen una hemoglobina glicosilada de $7.46(\mathrm{DE}=1.61)$, un colesterol total promedio de $205.23(D E=39,74)$; la media del colesterol LDL fue $118(D E=46.64)$ y HDL 49.28 (DE = 11.1); triglicéridos 175.6 (DE $=126.62)$. La talla y peso promedio fueron 1.68 $\mathrm{cm} .(\mathrm{DE}=0.85)$. La presión sistólica y diastólica promedió 137.26 $(\mathrm{DE}=17,53)$ y $76.78(\mathrm{DE}=$ 9.77), respectivamente. 59 pacientes $(77.6 \%)$ presentan comorbilidad con la hipertensión. Tan sólo 4 padece nefropatía, 12 retinopatía, en tanto que no se identifican casos de neuropatía. 39 pacientes presentan un buen control metabólico $(\mathrm{Hb} 1 \mathrm{Ac} \leq 7 \%)$.

\section{Instrumentos}

La Escala Multidimensional de Apoyo Social Percibido de Zimet et al. (1988), está conformada por 12 afirmaciones (ítems) que evalúan la percepción de apoyo social. Estos ítems se agrupan en 3 factores. El primero mide apoyo social de pares (ítems $6,7,9$ y 12), el segundo apoyo de la familia (ítems $3,4,8$ y 11) y el tercero percepción de apoyo de la pareja u otras figuras significativas (ítems 1, 2, 5 
y10). El formato de respuesta es de tipo Likert, de cuatro puntos $(1=$ casi siempre; $2=$ a veces; $3=$ con frecuencia; 4 = siempre o casi siempre). A mayor puntaje obtenido, se estima mayor percepción de apoyo social.

\section{Procedimiento de recolección de datos}

Los sujetos fueron contactados en la sala de espera del consultorio Miraflores de la ciudad de Temuco. Todos los pacientes firmaron una carta de consentimiento informado, en la cual se dio a conocer el objetivo del estudio, se explicitó la voluntariedad de la participación, así como el carácter anónimo y confidencial del uso de la información.

La recolección de datos, se efectuó en el mes en julio del año 2008. Para cautelar la comprensión de los participantes y calidad de la respuesta, tanto el consentimiento informado como el instrumento fueron aplicados directamente por estudiantes de último años de la carrera de Psicología de la Universidad de La Frontera, quienes previamente recibieron entrenamiento y capacitación para tal efecto.

Como marco regulatorio de la recolección de datos y para resguardar el bienestar de los participantes, se cumplieron los principios éticos que dicta la American Psychological Association (American Psychological Association, 1992).

\section{Análisis de los datos}

Los datos fueron analizados con el procesador estadístico SPSS 16.0 para MAC OS X 10.5.6. Se estimó la confiabilidad del instrumento por medio del cálculo del índice de consistencia interna alfa de Cronbach. Se realizó análisis factorial exploratorio, con extracción de Componentes Principales y rotación Varimax.

\section{Resultados}

\section{Confiabilidad del instrumento}

Para el cálculo de la confiabilidad del instrumento, se procedió a realizar análisis de confiabilidad alfa de Cronbach, para la totalidad de la escala. Tal como se observa en la Tabla 1, las correlaciones entre los ítems y el puntaje total son superiores a .03. A su vez, la confiabilidad total del instrumento es de .0849 , con la totalidad de los ítems. Paralelamente, se realizó el análisis de confiabilidad para cada uno de los factores que conforman la escala. En el caso del primer factor percepción de apoyo social de pares, la confiabilidad para las cuatro afirmaciones fue 0.873. Para el segundo factor percepción de apoyo de la familia, el coeficiente alfa de Cronbach fue de .823, y para el tercer factor apoyo social de otros significativos, el valor fue de .748 (véase Tabla 1).

TABLA 1

Correlación ítem total corregida y alfa de Cronbach si se elimina el ítem

\begin{tabular}{lcc}
\hline & $\begin{array}{c}\text { Correlación ítem- } \\
\text { total corregida }\end{array}$ & $\begin{array}{c}\text { Alfa de Cronbach si } \\
\text { se elimina el ítem }\end{array}$ \\
\hline Cuando necesito algo, sé que hay alguien que me puede ayudar & 0.434 & 0.843 \\
Cuando tengo penas o alegrías, hay alguien que me puede ayudar & 0.468 & 0.841 \\
Tengo la seguridad que mi familia trata de ayudarme & 0.534 & 0.840 \\
Mi familia me da la ayuda y apoyo emocional que requiero & 0.489 & 0.837 \\
Hay una persona que me ofrece consuelo cuando lo necesito & 0.587 & 0.840 \\
Tengo la seguridad de que mis amigos tratan de ayudarme & 0.596 & 0.833 \\
Puedo contar con mis amigos cuando tengo problemas & 0.832
\end{tabular}




\begin{tabular}{lcc}
\hline & $\begin{array}{c}\text { Correlación ítem- } \\
\text { total corregida }\end{array}$ & $\begin{array}{c}\text { Alfa de Cronbach si } \\
\text { se elimina el ítem }\end{array}$ \\
\hline Puedo conversar de mis problemas con mi familia & 0.536 & 0.837 \\
Cuando tengo alegrías o penas puedo compartirlas con mis amigos & .510 & .839 \\
Hay una persona que se interesa por lo que yo siento & 0.574 & 0.835 \\
Mi familia me ayuda a tomar decisiones & 0.516 & 0.839 \\
Alfa de Cronbach 12 ítems $=$ & 0.849 & \\
Alfa Escala Apoyo social pares $=$ & 0.873 & \\
Alfa Escala Apoyo social familia $=$ & 0.823 & \\
Alfa Escala Apoyo otros significativos $=$ & 0.748 & \\
\hline
\end{tabular}

Fuente: elaboración propia.

\section{Análisis factorial de la escala}

Para estimar la validez de la escala, se realizó un análisis factorial exploratorio. Tal como se aprecia en la Tabla 2, la medida de adecuación muestral de Kaiser Meyer-Olkin (KMO) resulta adecuada con un valor de 0.792. Asimismo, la prueba de esfericidad de Bartlett es significativa $\left(x^{2}=693.293\right.$; $p$ $\leq .001)$. Estos resultados indican que se cumplen con las condiciones necesarias para proseguir con el análisis factorial.

TABLA 2

Condiciones aplicación análisis factorial

\begin{tabular}{llr}
\hline \multicolumn{3}{c}{ KMO y prueba de Bartlett } \\
\hline KMO & & .792 \\
Prueba de esfericidad & $\begin{array}{l}\text { Chi-cuadrado } \\
\text { de Bartlett }\end{array}$ & 693.293 \\
& aproximado & 66 \\
& $g l$ & 0.000 \\
\hline
\end{tabular}

Fuente: elaboración propia.

En la Tabla 3, es posible apreciar, los factores que arroja el análisis de componentes principales, el porcentaje de varianza individual de cada uno y el porcentaje de varianza acumulada.

Consistente, con la estructura propuesta por Zimet et al. (1988), se extraen 3 factores con au- tovalores mayores a 1. El primer factor explica un $38.005 \%$ de la varianza, el segundo un $18.4 \%$ y el tercero un $10.45 \%$. En conjunto los tres factores dan cuenta de un $66.8 \%$ de la varianza total.

Si se observa la Figura 1, que corresponde al gráfico de sedimentación, el resultado anterior tiende a confirmarse.

\section{TABLA 3}

Autovalores y porcentaje de varianza que explica cada factor

\begin{tabular}{cccc}
\hline \multirow{2}{*}{$\begin{array}{c}\text { Compo- } \\
\text { nente }\end{array}$} & \multicolumn{3}{c}{ Autovalores iniciales } \\
\cline { 2 - 4 } & Total & \% de la varianza & $\%$ acumulado \\
\hline 1 & 4,561 & 38,005 & 38,005 \\
2 & 2,209 & 18,404 & 56,410 \\
3 & 1,255 & 10,455 & 66,865 \\
4 & 0,755 & 6,293 & 73,158 \\
5 & 0,658 & 5,480 & 78,638 \\
6 & 0,550 & 4,585 & 83,223 \\
7 & 0,510 & 4,251 & 87,475 \\
8 & 0,469 & 3,911 & 91,386 \\
9 & 0,357 & 2,971 & 94,357 \\
10 & 0,299 & 2,489 & 96,846 \\
11 & 0,208 & 1,735 & 98,581 \\
12 & 0,170 & 1,419 & 100,000 \\
\hline
\end{tabular}

Fuente: elaboración propia. 
Figura 1

Gráfico sedimentación Escala MSPSS

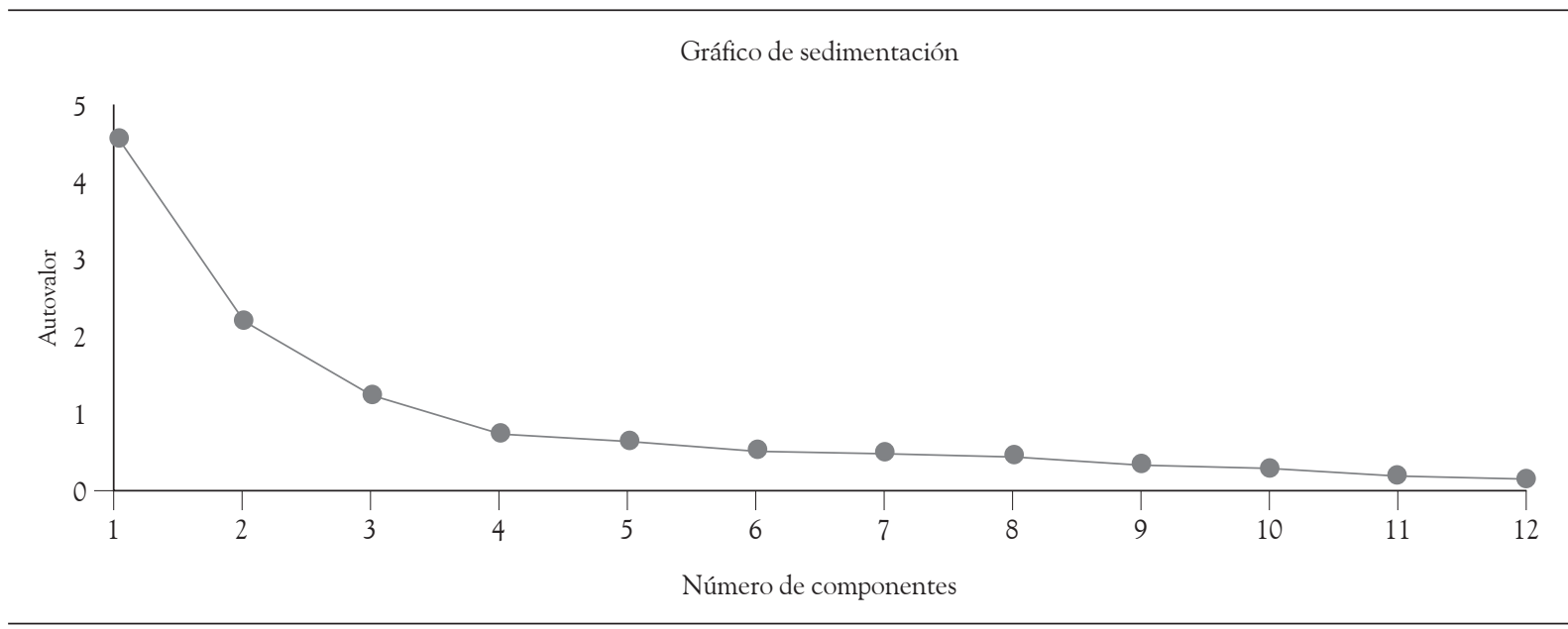

Fuente: elaboración propia.

En la Tabla 4, se puede apreciar la matriz de factores rotados con los valores de saturación de cada ítem en cada factor. El primer factor agrupa los ítems 6, 7, 9 y 12, con cargas factoriales que oscilan entre 0.792 y 0.860 . Este factor ha sido denominado apoyo social de pares o amigos. El segundo factor agrupa los ítems 3, 4, 8 y 11, todos relacionados con el apoyo social de la familia. Las cargas factoriales de estos ítems en el factor varían entre 0.632 y 0.892 . Finalmente, el tercer factor queda conformado por los ítems 1, 2, 5 y10, cuyas cargas factoriales oscilan entre 0.644 y 0.787 .

TABLA 4

Matriz de factores rotados, con valores que saturan en cada factor

\begin{tabular}{|c|c|c|c|}
\hline & $\begin{array}{c}\text { Factor 1: } \\
\text { Percepción apoyo } \\
\text { de pares }\end{array}$ & $\begin{array}{c}\text { Factor 2: } \\
\text { Percepción apoyo } \\
\text { de familia }\end{array}$ & $\begin{array}{c}\text { Factor 3: } \\
\text { Percepción apoyo } \\
\text { otros significativos }\end{array}$ \\
\hline Puedo conversar de mis problemas con mis amigos & 0.860 & & \\
\hline Puedo contar con mis amigos cuando tengo problemas & 0.851 & & \\
\hline $\begin{array}{l}\text { Cuando tengo alegrías o penas puedo compartirlas con } \\
\text { mis amigos }\end{array}$ & 0.817 & & \\
\hline $\begin{array}{l}\text { Tengo la seguridad de que mis amigos tratan de ayu- } \\
\text { darme }\end{array}$ & 0.792 & & \\
\hline $\begin{array}{l}\text { Mi familia me da la ayuda y apoyo emocional que } \\
\text { requiero }\end{array}$ & & 0.892 & \\
\hline Tengo la seguridad que mi familia trata de ayudarme & & 0.807 & \\
\hline Mi familia me ayuda a tomar decisiones & & 0.781 & \\
\hline Puedo conversar de mis problemas con mi familia & & 0.632 & \\
\hline
\end{tabular}




\begin{tabular}{|c|c|c|c|}
\hline & $\begin{array}{c}\text { Factor 1: } \\
\text { Percepción apoyo } \\
\text { de pares }\end{array}$ & $\begin{array}{l}\text { Factor 2: } \\
\text { Percepción apoyo } \\
\text { de familia }\end{array}$ & $\begin{array}{c}\text { Factor 3: } \\
\text { Percepción apoyo } \\
\text { otros significativos }\end{array}$ \\
\hline $\begin{array}{l}\text { Cuando necesito algo, sé que hay alguien que me pue- } \\
\text { de ayudar }\end{array}$ & & & 0.787 \\
\hline $\begin{array}{l}\text { Hay una persona que me ofrece consuelo cuando lo } \\
\text { necesito }\end{array}$ & & & 0.706 \\
\hline Hay una persona que se interesa por lo que yo siento & & & 0.704 \\
\hline $\begin{array}{l}\text { Cuando tengo penas o alegrías, hay alguien que me } \\
\text { puede ayudar }\end{array}$ & & & 0.644 \\
\hline
\end{tabular}

Fuente: elaboración propia.

\section{Discusión}

El objetivo de este estudio fue estimar las propiedades psicométricas de la escala de Zimet at al. (1989) que evalúa la percepción de apoyo social. En relación con la consistencia interna de la escala, ésta parece adecuada, presentando valores por sobre 0.8 para los factores de percepción de apoyo del grupo de pares y de la familia, y superior a 0.7 para la escala de otros significativos. La consistencia interna de los 12 reactivos es cercana a 0.85 , lo que analizado en su conjunto indica que se está en presencia de una escala confiable.

En su versión original la escala está conformada por tres factores que permiten dimensionar tres fuentes de apoyo social: la familia, los pares y otros significativos. Al respecto, en esta investigación se confirma la estructura propuesta por los autores, al identificarse claramente los tres factores mencionados, cada uno de ellos conformado por 4 ítems, y responsables en su conjunto del $66.8 \%$ de la varianza. Las cargas factoriales de cada ítem en cada factor son todas superiores a 0.6.

En tal sentido, la escala MSPSS posee en buen comportamiento psicométrico, presentando evidencia de una adecuada validez factorial y buenos indicadores de confiabilidad. Si a esto se suma que la escala es breve, tan sólo 12 ítems, de fácil administración y puntuación, parece que la escala MSPSS resulta propicia para su utilización en población de pacientes con enfermedades crónicas, en especial pacientes con DM2.
Sin embargo, pese a estos hallazgos, resulta interesante discutir la diferencia que podría establecerse entre la percepción de apoyo social de la familia y de otras figuras significativas. Esto si se toma en consideración que en un estudio de validación de esta misma escala realizado por (Arechabala \& Miranda, 2002), en población de pacientes hipertensos chilenos, se identificaron tan solo 2 de los 3 factores propuestos por Zimet et al. (1988), agrupándose los ítems del factor otros significativos mayoritariamente en el factor de percepción de apoyo familiar. Es interesante cuestionarse si la población de pacientes DM2 encuestada, diferencia claramente estas dos fuentes de apoyo social, pues, por ejemplo, al aplicar el ítem "hay una persona que me ofrece consuelo cuando lo necesito" (reactivo perteneciente a la escala de otros significativos) frecuentemente los participantes referían que esa otra persona, era una figura religiosa y no al real significado que los autores le imprimen al factor “otros significativos”, tal como es comprendido en población norteamericana.

Otro aspecto que podría ser considerado en la estimación de la validez de constructo, con alguna otra variable que teóricamente se encuentre relacionada, tal como el nivel de estrés, depresión o calidad de vida. Al respecto existe evidencia que hace suponer que estos constructos están teóricamente asociados (Coffman, 2008; Göz et al., 2007; Sacco \& Yanover, 2006).

Por último, con miras a agregar otra evidencia de las propiedades psicométricas de esta escala, 
se podría calcular la confiabilidad test-retest, con la finalidad de estimar la estabilidad temporal del instrumento.

\section{Referencias}

Arechabala, M. \& Miranda, C. (2002). Validación de una escala de apoyo social percibido en un grupo de adultos mayores adscritos a un programa de hipertensión de la Región Metropolitana. Ciencia y Enfermería, 8(1), 49-55.

American Psychological Association. (1992). Ethical principles of psychologists and code of conduct. American Psychologist, 47, 1597-1611.

Barrera, M. \& Ainlay, S. L. (1983). The structure of social support: A conceptual and empirical analysis. Journal of Community Psychology, 1, 133-143.

Calvo Francés, F. \& Díaz Palarea, M. (2004). Apoyo social percibido: características psicométricas del cuestionario Caspe en una población urbana geriátrica. Psicothema, 16(4), 570-575.

Canty-Mitchell, J. \& Zimet, G. (2000). Psychometric properties of the Multidimensional Scale of Perceived Social Support in urban adolescents. American Journal of Community Psychology, 28, 391-400.

Coffman, M. J. (2008). Effects of tangible social support and depression on diabetes self-efficacy. Journal of Gerontological Nursing, 34(4), 32-39.

Dahlem, N., Zimet, G. \& Walker, R. (1991). The Multidimensional Scale of Perceived Social Support: a confirmation study. Journal of Clinical Psychology, 47, 756-761.

Di Matteo, M. R. (2004). Social support and patient adherence to medical treatment: A meta-analysis. Health Psychology, 23(2), 207-218.

Göz, F., Karaoz, S., Goz, M., Ekiz, S. \& Cetin, I. (2007). Effects of the diabetic patient`s perceived social support on their quality-of-life. Journal of Clinical Nursing, 16, 1353-1360.

Holguín, L., Correa, D., Arrivillaga, M., Cáceres, D. \& Varela, M. (2006). Adherencia al tratamiento de hipertensión arterial: efectividad de un programa de intervención biopsicosocial. Universitas Psychologica, 5(3), 533-547.
Kazarian, S. \& McCabe, S. (1991). Dimensions of social support in the MSPSS: Factorial structure, reliability, and theoretical implications. Journal of Community Psychology, 19, 150-160.

Kyngäs, H. (2000). Compliance of adolescents with diabetes. Internacional Pediatrics Nursing, 15, 260-267.

Ortiz, M. (2004). Factores psicológicos y sociales asociados a la adherencia al tratamiento en adolescentes diabéticos tipo 1. Psykhe, 13(1), 21-31.

Ortiz, M. \& Ortiz, E. (2007). Psicología de la salud: una clave para comprender el fenómeno de la adherencia terapéutica. Revista Médica de Chile, 135, 647-652.

Passa, P. (2002). Diabetes trends in Europe. Diabetes/ Metabolism Research and Reviews, 18 (Suppl. 3), S3-S8.

Quirós-Morales, D. \& Villalobos-Pérez, A. (2007). Comparación de factores vinculados a la adherencia al tratamiento en Diabetes Mellitus tipo II entre una muestra urbana y otra rural de Costa Rica. Universitas Psychologica, 6(3), 679-688.

Sacco, W. P. \& Yanover, T. (2006). Diabetes and depression: The role of social support and medical symptoms. Journal of Behavioral Medicine, 29(6), 523-531.

Sarason, I. G. (1983). Assessing social support: The Social Support Questionary. Journal of Personality and Social Psychology, 44, 127-139.

Sherbourne, C. \& Stewart, A. (1991). The MOS Social Support Survey. Social Science \& Medicine, 32, 705-714.

Westaway, M. S., Seager, J. R., Rheeder, P. \& Van Zyl, D. G. (2005). The effects of social support on health, well-being and management of Diabetes Mellitus: A black South African perspective. Ethnicity $\mathcal{E}$ Health, 10(1), 73-89.

Zhang, X., Norris, S. L., Gregg, E. W. \& Beckles, G. (2007). Social support and mortality among older persons with diabetes. The Diabetes Educator, 33(2), 273-281.

Zimet, G., Dahlem, N., Zimet, S. \& Farley, G. (1988). The Multidimensional Scale of Perceived Social Support. Journal of Personality Assessment, 52, $30-41$. 